\title{
CERTAIN CONTACT PROPERTIES OF LINEAR SYSTEMS OF HYPERSURFACES*
}

BY

B. C. WONG

Introduction. The equation

$$
\xi_{0} f^{(0)}+\xi_{1} f^{(1)}+\cdots+\xi_{\rho} f^{(p)}=0,
$$

where the $\xi$ 's are homogeneous parameters and the $f$ 's are homogeneous functions of degree $n$ in the variables $x_{0}, x_{1}, \cdots, x_{r}$, represents an $\infty$-system, $|V|$, of hypersurfaces of order $n$ in an $r$-space, $S_{r}$. Interpreting the $\xi$ 's as the homogeneous coordinates of a point of a $\rho$-space, $\Sigma_{\rho}$, we have a one-to-one correspondence between the points of $\Sigma_{\rho}$ and the hypersurfaces of $|V|$.

In this paper we propose to deal with certain contact properties of the system $|V|$ and to investigate some of the properties and relations to one another of the corresponding loci in $\Sigma_{\rho}$. Our results will be generalizations of certain results which W. L. Edge thas, in connection with his study of octadic surfaces, described for the case $r=3, \rho=2, n=2$. We should mention that T. R. Hollcroft has derived $\ddagger$ the properties of the curve in the plane $\Sigma_{2}$ of the parameters which corresponds to the Jacobian curve of a net of hypersurfaces in $S_{r}$, and in another paperई those of the surface in the 3-dimensional space $\Sigma_{3}$ of the parameters which corresponds to the Jacobian surface of a web of surfaces in $S_{3}$. In neither of these papers, however, has the author touched upon the results which we are going to derive.

In the following we shall, first, describe the general case. This is done in $\$ 1$. Then we shall consider the case $r=\rho=2$ in $\$ 2$ and the case $n=2, r=\rho$ general in $\S 3$. Finally, in $\$ 4$, we shall conclude the paper with a description of the results for $n=2, r=\rho=3$.

1 . The general case. Let $n, r, \rho$ be general. Since for a hypersurface to acquire a conical point or hypernode is equivalent to one condition, there are $\infty^{p-1}$ hypersurfaces of the system $|V|$ each possessing a hypernode. The

* Presented to the Society, June 20,1934, under a slightly different title; received by the editors May 2, 1934.

† Octadic surfaces and plane quartic curves, Proceedings of the London Mathematical Society, (2), vol. 34 (1932), pp. 492-525.

$\ddagger$ Nets of manifolds in i dimensions, Annali di Matematica, (4), vol. 5 (1927-1928), pp. 261-267.

$\$$ The general web of algebraic surfaces of order $n$ and the involution defined by it, these Trangactions, vol. 35 (1933), pp. 855-868. 
locus of these hypernodes is a $(\rho-1)$-dimensional manifold, the Jacobian manifold, $J_{p-1}^{m}$, of $|V|$. Differentiating equation (1) partially with respect to $x_{i}$, we have, writing $f_{i}$ for $\partial f / \partial x_{i}$,

$$
\xi_{0} f_{i}^{(0)}+\xi_{1} f_{i}^{(1)}+\cdots+\xi_{\rho} f_{i}^{(\rho)}=0 \quad[i=0,1, \cdots, r] .
$$

The result of eliminating the $\xi$ 's from these equations is

$$
\left\|\begin{array}{cccc}
f_{0}^{(0)} & f_{1}^{(1)} & \cdots & f_{r}^{(r)} \\
f_{0}^{(1)} & f_{1}^{(1)} & \cdots & f_{r}^{(1)} \\
\cdot \cdot & \cdot & \cdot & \cdot \\
f_{0}^{(\rho)} & f_{1}^{(\rho)} & \cdots & f_{r}^{(p)}
\end{array}\right\|=0,
$$

which are the equations of $J_{\rho-1}^{m}$. To determine $m$, notice that the matrix in (3) contains $\rho+1$ rows and $r+1$ columns. Then the system of equations given by (3) is a restricted system of equations and its order is, the elements of the matrix being all of order $n-1, *$

$$
m=\left(\begin{array}{c}
r+1 \\
r-\rho+1
\end{array}\right)(n-1)^{r-p+1} .
$$

Now if we eliminate the $x$ 's from (2), we obtain a result in the form

$$
\Delta\left(\xi_{0}, \xi_{1}, \cdots, \xi_{\rho}\right)=0
$$

which is of degree $\dagger$

$$
\mu=(r+1)(n-1)^{r}
$$

and therefore represents a hypersurface $\Delta_{\rho-1}^{\mu}$ of order $\mu$ in the $\rho$-space $\Sigma_{\rho}$. The points of this hypersurface give the hypersurfaces of $|V|$ that have each a hypernode and may be said to correspond one-to-one to the points of $J_{p-1}^{m}$.

Let us now consider a variety $V_{k}^{N}$ of $k$ dimensions in $S_{r}$ and let it be the complete intersection of $r-k$ hypersurfaces of orders $n_{1}, n_{2}, \cdots, n_{r-k}$ given by the equations

$$
F^{(1)}=0, F^{(2)}=0, \cdots, F^{(r-k)}=0
$$

respectively. Then $N=n_{1} n_{2} \cdots n_{r-k}$. Contact being one condition, there are $\infty^{p-1}$ hypersurfaces of $|V|$ tangent to $V_{k}^{N}$ and the locus of the points of con-

* Salmon, Modern Higher Algebra, 4th edition, Lesson 19.

† The method of deriving this result is analogous to or an extension of the one described by Salmon in his Analytic Geometry of Three Dimensions, 5th edition, vol. 2, Art. 576. 
tact is $V_{k}^{N}$ itself if $k<\rho$ and a $V_{\rho-1}^{N^{\prime}}$ if $k \geqq \rho$. The equations of $V_{\rho-1}^{N^{\prime}}$ are the equations (4) and

$$
M \equiv\left\|\begin{array}{llll}
f_{0}^{(0)} & f_{1}^{(0)} & \cdots & f_{r}^{(0)} \\
f_{0}^{(1)} & f_{1}^{(1)} & \cdots & f_{r}^{(1)} \\
\cdot \cdot \cdot & \cdot \cdot \cdot \cdot \cdot \cdot \cdot \cdot \cdot \cdot \cdot f^{(\rho)} \\
f_{0}^{(\rho)} & f_{1}^{(\rho)} & \cdots & f_{r}^{(1)} \\
F_{0}^{(1)} & F_{1}^{(1)} & \cdots & F_{r}^{(1)} \\
\cdot \cdot \cdot & \cdot \cdot \cdot \cdot \cdot \cdot \cdot \cdot \cdot \\
F_{0}^{(r-k)} & F_{1}^{(r-k)} & \cdots & F_{r}^{(r-k)}
\end{array}\right\|=0
$$

that is, $V_{\rho-1}^{N^{\prime}}$ is the intersection of $V_{k}^{N}$ and the Jacobian manifold of the hypersurfaces given by (4) and any $\rho+1$ independent hypersurfaces of $|V|$. In the above we have written $F_{i}$ for $\partial F / \partial x_{i}$.

Now to determine the order $N^{\prime}$ of $V_{\rho-1}^{N \prime}$, it is necessary first to determine the order of the restricted system of equations $M=0$. We see that $M$ contains $r-k+\rho+1$ rows and $r+1$ columns and that the elements $f_{i}$ are all of order $n-1$ and $F_{i}^{(j)}$ are of order $n_{j}-1$. Therefore, the order of $M$ is*

$$
\sum_{i=0}^{k-p+1} C_{k-\rho+1-i} H_{i}
$$

where

$$
\begin{aligned}
C_{0} & =H_{0}=1 \\
C_{k-\rho+1-i} & =\left(\begin{array}{c}
r+1 \\
k-\rho+1-i
\end{array}\right)(n-1)^{k-\rho+1-i}, \\
H_{i} & =\sum_{q} \sum\left(n_{1}-n\right)^{q 1}\left(n_{2}-n\right)^{q 2} \cdots\left(n_{i}-n\right)^{q i}
\end{aligned}
$$

where

$$
q=q_{1}+q_{2}+\cdots+q_{i}=i .
$$

Therefore, the order of $V_{\rho-1}^{N^{\prime}}$ is $N$ times the order of $M$, that is,

$$
N^{\prime}=n_{1} n_{2} \cdots n_{r-k} \sum_{i=0}^{k-\rho+1} C_{k-\rho+1-i} H_{i} .
$$

Correspondingly, we find that there is a hypersurface $\Phi_{\rho-1}^{\nu}$ of order $\nu$ in $\Sigma_{\rho}$ whose points yield the hypersurfaces of $|V|$ tangent to $V_{k}^{N}$ and may be

* Salmon, Modern Bigher Algebra, 4th edition, Lesson 19. 
said to correspond to the points of $V_{\rho-1}^{N^{\prime}}$. The condition of contact between a hypersurface of $|V|$ and $V_{k}^{N}$ is the vanishing of the tact-invariant which involves the coefficients in (1) in the degree $\nu$. We have, then, ${ }^{*}$

$$
\nu=n_{1} n_{2} \cdots n_{r-k} \sum_{i=0}^{k}\left(\begin{array}{c}
r+1 \\
k-i
\end{array}\right) H_{i} .
$$

For $k<\rho$, the hypersurface $\Phi_{\rho^{\prime}-1}$ is the locus of $\infty^{k}(\rho-k-1)$-spaces and, for $k \geqq \rho$, it is a point locus. As our knowledge of hypersurfaces is still very scanty, we find it difficult to describe the $\Phi_{\rho_{-1}{ }^{p}}$ 's for all values of $k<r$ and their relations to one another. We shall, however, content ourselves with a few special cases.

2. The case $r=\rho=2$. Now we have a net $|c|$ of curves of order $n$ in an $x$-plane to which correspond the points of a $\xi$-plane. The Jacobian curve $J \mathbf{m}$ of $|c|$ is of order $m=3(n-1)$ and the corresponding curve $\Delta^{\mu}$ in the $\xi$-plane is of order $\mu=3(n-1)^{2}$. The characteristics of this curve $\Delta^{\mu}$ are known. $\dagger$

Now consider a curve $C^{n_{1}}$ of order $n_{1}$ in the $x$-plane. Here $k=1$. From result (5) we have $\nu=n_{1}\left(n_{1}+2 n-3\right)$ for the order of the curve $\Gamma^{v}$ in the $\xi$-plane whose points give the curves of the net tangent to $C^{n_{1}}$. If $C^{n_{1}}$ has $d_{1}$ nodes and $k_{1}$ cusps, the quantity $2 d_{1}+3 k_{1}$ is to be deducted from the above result. Since the points of $\Gamma^{v}$ and the points of $C^{n_{1}}$ are in one-to-one correspondence, the genus of $\Gamma^{v}$ is

$$
\begin{aligned}
p & =\frac{1}{2}(\nu-1)(\nu-2)-\delta-\kappa \\
& =\frac{1}{2}\left(n_{1}-1\right)\left(n_{1}-2\right)-d_{1}-k_{1},
\end{aligned}
$$

where $\delta$ is the number of nodes on $\Gamma^{v}$ and is equal to the number of the curves of $|c|$ doubly tangent to $C^{n_{1}}$, and $\kappa$ is the number of cusps on $\Gamma^{\nu}$ and is equal to the number of the curves of $|c|$ having each a 3-point contact with $C^{n_{1}}$. Hence, the characteristics of $\Gamma^{\nu}$ are

$$
\begin{aligned}
\nu & =n_{1}\left(n_{1}+2 n-3\right)-2 d_{1}-3 k_{1}, \ddagger \\
\delta & =\frac{1}{2} \nu(\nu-9)-\frac{1}{2} n_{1}\left(n_{1}-9\right)+3 n_{1}(n-1)+d_{1}, \\
\kappa & =3 n_{1}\left(n+n_{1}-3\right)-6 d_{1}-8 k_{1}, \\
\nu^{\prime} & =n_{1} n,
\end{aligned}
$$

the last being the class of the curve.

\footnotetext{
- Salmon, Analytic Geometry of Three Dimensions, above.

$\dagger$ See Hollcroft, Nets of manifolds in $i$ dimensions, above.

¥ This formula is given in Salmon, Higher Plane Curves, 3d edition, Art. 97.
} 
Since the given curve $C^{n_{1}}$ meets $J^{m}$ in $n_{1} m=3 n_{1}(n-1)$ points, the curve $\Gamma^{*}$ touches the corresponding curve $3 n_{1}(n-1)$ times and meets it again in $\mu \nu-6 n_{1}(n-1)=3 n_{1}(n-1)\left(n_{1} n+2 n^{2}-5 n-n_{1}+1\right)$ points. This is the number of the curves of $|c|$ which possess each a node and are tangent to $C^{n_{1}}$. $\Gamma^{*}$ touching $\Delta^{\mu} 3 n_{1}(n-1)$ times and possessing $\delta$ nodes and $\kappa$ cusps must satisfy $3 n_{1}(n-1)+\delta+2 \kappa$ conditions and the degree of its freedom is $\frac{1}{2} \nu(\nu+3)$ $-3 n_{1}(n-1)-\delta-2 \kappa$ which is equal to $\frac{1}{2} n_{1}\left(n_{1}+3\right)-d_{1}-2 k_{1}$, the degree of freedom of $C^{n_{1}}$ in the $x$-plane. Hence, we have a one-to-one correspondence between the curves of the type $\Gamma^{\nu}$ in the $\xi$-plane and all the curves of order $n_{1}$ for all values of $n_{1}$ in the $x$-plane.

For $n=r=\rho=2$, we have a net of conics in a plane, the $x$-plane. Corresponding to the conics of this net are the points of another plane, the $\xi$-plane. In the $x$-plane there is the Jacobian curve, $J^{3}$, the locus of the vertices of the degenerate conics of the net, and in the $\xi$-plane there is a cubic curve, $\Delta^{3}$, whose points correspond to the degenerate conics of the net or may be said to correspond to the points of the Jacobian $J^{3}$. The conics of the net which are tangent to a given line $l$ of the $x$-plane are given by the points of a conic $\lambda^{2}$ in the $\xi$-plane. This conic $\lambda^{2}$ is triply tangent to $\Delta^{3}$, the points of contact being the images of the points on $J^{3}$ in which $l$ intersects it. Thus, we have a one-to-one correspondence between the lines of the $x$-plane and the conics triply tangent to $\Delta^{3}$ in the $\xi$-plane. Now through a given point $A$ of the $x$ plane pass the conics of a pencil belonging to the net and in the $\xi$-plane we have a line $\alpha$. Then, a one-to-one correspondence exists between the points of the $x$-plane and the lines of the $\xi$-plane. If $A$ is on $l$, then $\alpha$ is tangent to $\lambda^{2}$ at the point which corresponds to the conic of the net tangent to $l$ at $A$. We may look for loci of points in the $\xi$-plane which yield conics tangent to given curves in the $x$-plane and describe their properties and their relations to one another and to the curve $\Delta^{3}$. But we shall not unnecessarily lengthen our work by a discussion of these details here as they can be easily obtained from the results above.

3. The case $n=2, r=\rho$ general. In this case we have an $\infty^{r}$-system of $|Q|$ of quadric hypersurfaces in an $r$-space $S_{r}$ and correspondingly the points of another $r$-space $\Sigma_{r}$. The Jacobian of $|Q|$ is a hypersurface $J_{r-1}^{r+1}$ of order $r+1$ in $S_{r}$ and the locus of points in $\Sigma_{r}$ corresponding to $J_{r-1}^{r+1}$ is a hypersurface $\Delta_{r-1}^{r+1}$ also of order $r+1$.

Let now an $(r-t)$-space, $S_{r-t}$, be given in $S_{r}$ and let its equation be

$$
u_{0}^{(j)} x_{0}+u_{1}^{(j)} x_{1}+\cdots+u_{r}^{(j)} x_{r}=0 \quad[j=1,2, \cdots, t] .
$$

The condition that a quadric hypersurface of $|Q|$ be tangent to $S_{r-t}$ is 


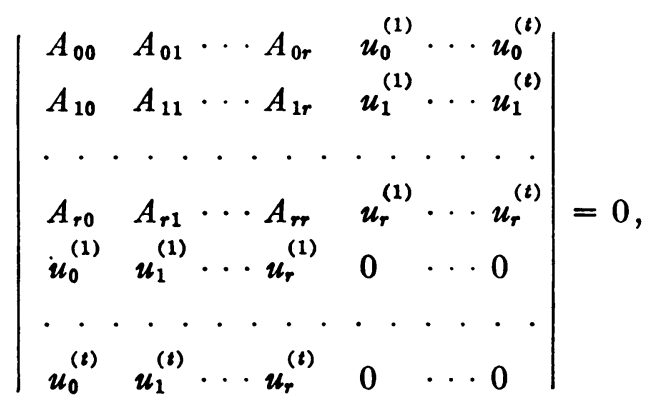

where $A_{i j}=a_{i j}{ }^{(0)} \xi_{0}+a_{i j}{ }^{(1)} \xi_{1}+\cdots+a_{i j}{ }^{(r)} \xi_{r}, a_{i j}{ }^{\left({ }^{(}\right)}$being the coefficients in the equation $f^{(h)}=0$ of the $h$ th quadric hypersurface determining the system $|Q|$. The equation above is that of a hypersurface $\Phi_{r-1}^{r-t+1}$ of order $r-t+1$ in $\Sigma_{r}$ whose points correspond to the quadric hypersurfaces of $|Q|$ tangent to $S_{r-t}$. We may say that there is a one-to-one correspondence between the points of $S_{r-t}$ and the points of $\Phi_{r-1}^{r-t+1}$.

Suppose $t=1$. Then, to all the $\infty^{r+1}$ hypersurfaces of $S_{r}$ correspond $\infty^{r+1}$ hypersurfaces of the type $\Phi_{r-1}^{r}$ in $\Sigma_{r}$. Each such $\Phi_{r_{-1}^{r}}^{r}$ is tangent to $\Delta_{r-1}^{r+1}$ along an $(r-2)$-dimensional variety, $\Theta_{r-2}^{r(r+1) / 2}$, of order $r(r+1) / 2$, and the points of this contact variety correspond to the points of the section of $J_{r-1}^{r+1}$ by the corresponding hyperplane $S_{r-1}$.

Now suppose $t=2$ and let $S_{r-2}$ be in a fixed $S_{r-1}$. The locus of points in $\Sigma_{r}$ corresponding to those quadric hypersurfaces of $|Q|$ tangent to $S_{r-2}$ is a hypersurface $\Phi_{r-1}^{r-1}$ of order $r-1$. This $\Phi_{r-1}^{r-1}$ is tangent to $\Delta_{r-1}^{r}$ corresponding to the fixed $S_{r-1}$ along an $(r-2)$-dimensional variety $\Theta_{r-2}^{r(r-1) / 2}$ and tangent to $\Delta_{r-1}^{r+1}$ along an $(r-3)$-dimensional variety $\Theta_{r-3}^{r(r+1)(r-1) / 6}$. The points of $\Theta_{r-2}^{r(r-1) / 2}$ correspond to the quadric hypersurfaces of $|Q|$ tangent to $S_{r-1}$ at the points of $S_{r-2}$. The points of $S_{r-2}$ considered as points of $S_{r-1}$ have for corresponding points the points of $\Theta_{r-2}^{r(r-1) / 2}$ lying on $\Phi_{r-1}^{r}$. The points of $S_{r-2}$ considered as points of contact between $S_{r-2}$ and those quadric hypersurfaces of $|Q|$ which are tangent to it without being tangent to $S_{r-1}$ have for corresponding points the points of $\Phi_{r-1}^{r-1}$. Now $S_{r-2}$ meets $J_{r-1}^{r+1}$ in a $V_{r-3}^{r+1}$ to which correspond the points of $\Theta_{r-3}^{r(r+1)(r-1) / 6}$.

Let us now consider a series of sub-spaces $S_{r-1}, S_{r-2}, \cdots, S_{r-t}, \cdots, S_{\mathbf{z}}$, $S_{1}, S_{0}$, each being contained in the preceding one. Then we have a series of hypersurfaces in $\Sigma_{r}$,

$$
\Phi_{r-1}^{r}, \Phi_{r-1}^{r-1}, \cdots, \Phi_{r-1}^{r-t+1}, \cdots, \Phi_{r-1}^{3}, \Phi_{r-1}^{2}, \Phi_{r-1}^{1}
$$

[the last one being a hyperplane] corresponding to the given sub-spaces respectively. We see that 


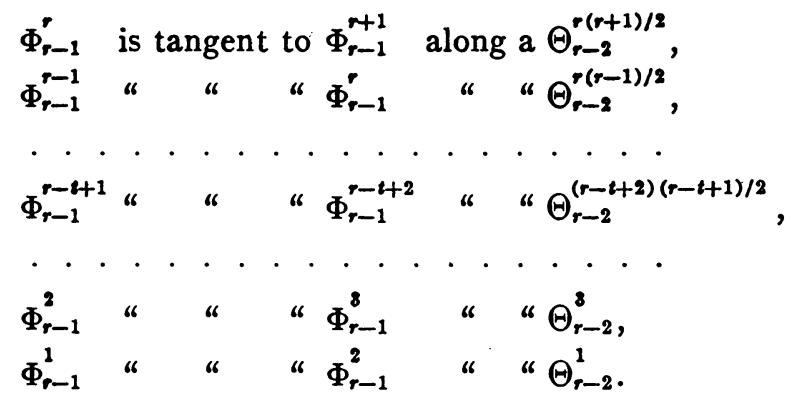

A general plane in $\Sigma_{r}$ meets $\Delta_{r-1}^{r+1}$ and all the $\Phi$ 's in a series of curves, an $(r+1)$-ic, an $r$-ic, $\cdots$, a cubic, a conic, and a line. The $(r+1)$-ic is touched by the $r$-ic at $r(r+1) / 2$ points, the $r$-ic by the $(r-1)$-ic at $r(r-1) / 2$ points, $\cdots$, the cubic by the conic at 3 points, and, finally, the conic is touched by the line. All these different groups of points of contact are the intersections of the plane and the various $\Theta_{r-2}$ 's.

Now

$$
\begin{aligned}
& \Theta_{r-2}^{r(r-1) / 2} \text { is tangent to } \Theta_{r-2}^{r(r+1) / 2} \text { along a } \Theta_{r-3}^{r(r+1)(r-1) / 8} \text {, } \\
& \Theta_{r-2}^{(r-1)(r-2) / 2} \text { " } \quad \text { " } \Theta_{r-2}^{r(r-1) / 2} \text { «" } \Theta_{r-3}^{r(r-1)(r-2) / 6},
\end{aligned}
$$

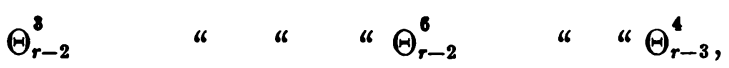

$$
\begin{aligned}
& \Theta_{r-2}^{1} \quad \text { " } \quad \text { " } \Theta_{r-2}^{3} \text { « } \quad \Theta_{r-3}^{1} \text {. }
\end{aligned}
$$

A general 3-space in $\Sigma_{r}$ meets the whole configuration consisting of $\Delta_{r-1}^{r+1}$ and the $\Phi$ 's in a configuration consisting of a series of surfaces $F^{r+1}, F^{r}, \cdots$, $F^{3}, F^{2}$, and a plane. The plane is tangent to $F^{2}$ which is a quadric cone along a line; $F^{2}$ is tangent to $F^{3}$ which is a four-nodal cubic surface along a twisted cubic curve $C^{3} ; F^{3}$ is tangent to $F^{4}$, a quartic symmetroid known to have ten conical points, along a sextic curve $C^{6}$ of genus $3 ; \cdots ; F^{r}$ is tangent to $F^{r+1}$ along a curve $C^{r(r+1) / 2}$. These curves of contact are the intersections of the $\Theta_{r-2}$ 's by the given 3-space. Now the line of contact between the plane and $F^{2}$ is tangent to $C^{3}$ at one point, $C^{3}$ is tangent to $C^{6}$ at 4 points, $C^{6}$ is tangent to $C^{10}$ at 10 points, $\cdots, C^{r(r-1) / 2}$ is tangent to $C^{r(r+1) / 2}$ at $r(r+1)(r-1) / 6$ points. These points of contact are the points where the given 3-space meets the various $\Theta_{r-3}$ 's.

We may continue this process until it terminates. But instead we shall exhibit the series of $\Phi$ 's and the different series of $\Theta$ 's in the following table, where for the moment (to simplify typography) we have set 


$$
(m, n)=\left(\begin{array}{l}
m \\
n
\end{array}\right) .
$$

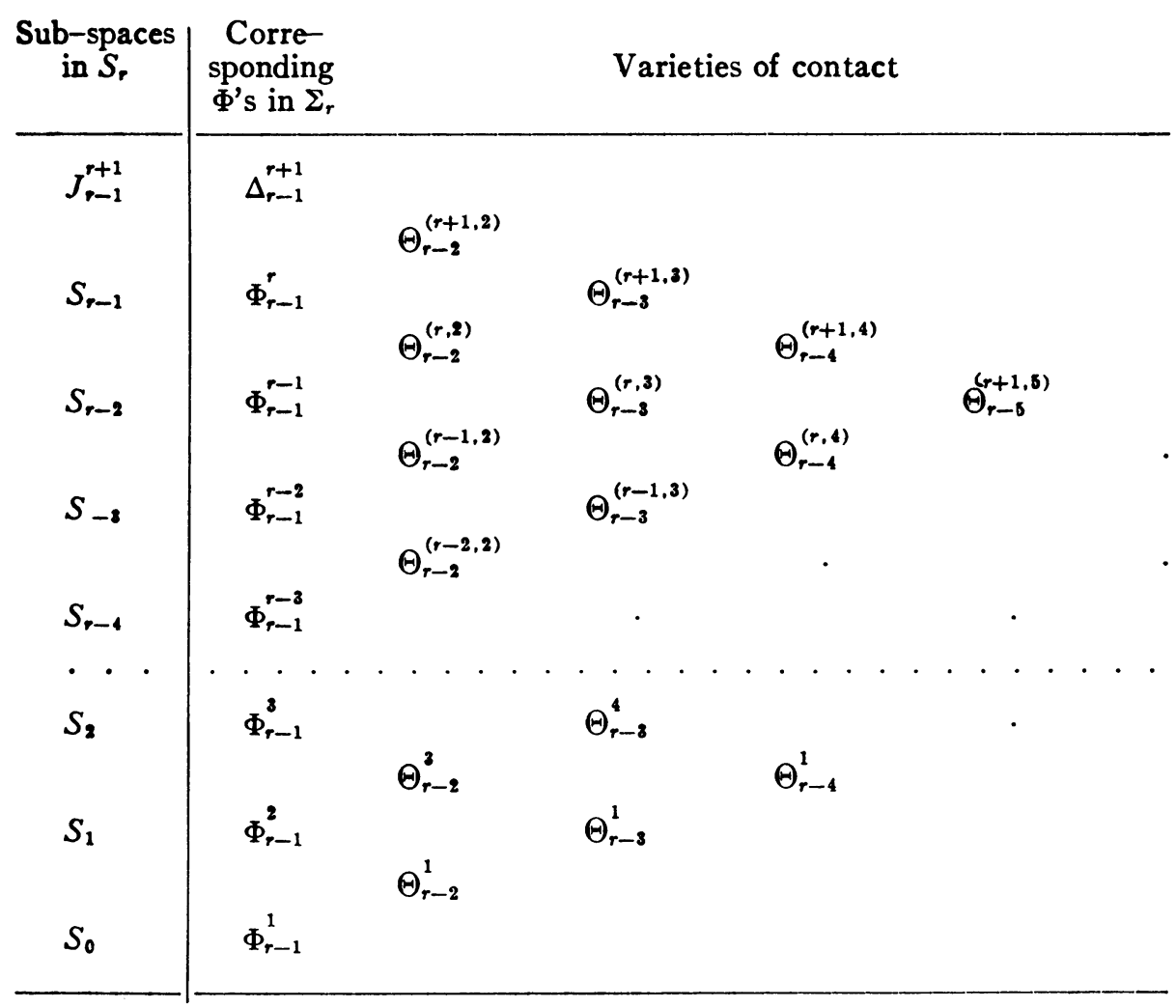

In this table any two symbols in the same column (other than the first) represent two varieties which touch along a variety whose symbol stands in the two diagonals in which the two symbols of the touching varieties lie. For example, $\Phi_{r-1}^{r}$ and $\Phi_{r-1}^{r-3}$ touch along $\Theta_{r-4}^{r(r-1)(r-2)(r-3) / 24}$ along which $\Theta_{r-2}^{r(r-1) / 2}$ and $\Theta_{r-2}^{(r-1)(r-2) / 2}$, and also $\Theta_{r-2}^{r(r-1)(r-2) / 6}$ and $\Theta_{r-3}^{(r-1)(r-2)(r-3) / 6}$ touch.

4. The case $n=2, r=\rho=3$. We have now a web of quadric surfaces in $S_{3}$ whose Jacobian is a quartic surface $J_{2}{ }^{4}$. The locus of points in $\Sigma_{3}$ corresponding to $J_{2}{ }^{4}$ is a quartic symmetroid $\Delta_{2}{ }^{4}$. A plane $f$ in $S_{3}$ meets $J_{2}{ }^{4}$ in a quartic curve $c^{4}$. The points of $\Sigma_{3}$ corresponding to those quadric surfaces of the web tangent to $f$ or to the points of $f$ as points of contact form a four-nodal cubic surface $F^{3}$ which is tangent to $\Delta_{2}{ }^{4}$ along a sextic curve $\Theta_{1}{ }^{6}$. $\Theta_{1}{ }^{6}$ passes through the four conical points of $F^{3}$ and its points correspond to the points of $c^{4}$. We have in $\Sigma_{3} \infty^{3}$ four-nodal cubic surfaces each having its four nodes lying on $\Delta_{2}^{4}$ and touching $\Delta_{2}^{4}$ along a sextic curve of genus 3 . 
Now take a line $l$ in $f$. The locus of points in $\Sigma_{3}$ corresponding to the quadric surfaces of the web tangent to $l$ or to the points of $l$ as points of contact is a quadric cone $F^{2}$. The vertex of $F^{2}$ corresponds to the quadric surface of the web passing through $l$ and it lies on $F^{3}$ corresponding to $f$. The line $l$ considered as a line of $f$ has for image a cubic curve $\Theta_{1}{ }^{3}$ on $F^{3} . F^{2}$ and $F^{3}$ touch along $\Theta_{1}{ }^{3}$. Now $\Theta_{1}{ }^{3}$ and the curve $\Theta_{1}{ }^{6}$ corresponding to $c^{4}$ common to $J_{2}{ }^{4}$ and $f$ are tangent at 4 points at which $F^{2}$ and $\Delta_{2}{ }^{4}$ are also tangent. These four points correspond to the four points common to $c^{4}$ and $l$ in $f . F^{2}$ intersects $\Delta_{2}^{4}$ in an octavic curve with four actual double points and the points of this curve give the cones of the web tangent to $l$. The locus of the vertices of these cones is a curve of order 12 on $J_{2}{ }^{4}$ and it also has four actual double points. We see that there are $\infty^{2}$ quadric cones in $\Sigma_{3}$ corresponding to the $\infty^{2}$ lines of $f$ each of which has its vertex on $F^{3}$ and is tangent to $F^{3}$ along a twisted cubic curve passing through the four nodes of $F^{3}$. Each point of $F^{3}$ is the vertex of two such cones, for a quadric surface of the web containing a line of $f$ contains another line of $f$.

If we take any curve, say a conic, $c^{2}$, in $f$, then the quadric surfaces of the web tangent to $c^{2}$ are given by the points of a ruled sextic surface $F^{6}$ in $\Sigma_{3}$. This surface $F^{6}$ is, in fact, a developable surface and it is tangent to $F^{3}$ along a sextic curve $\gamma^{6}$ whose points yield the quadric surfaces of the web tangent to $f$ with the points of contact on $c^{2}$. Its edge of regression is also a sextic curve and the nodal curve is a quartic, the points on the former giving the quadric surfaces which osculate $c^{2}$ and the points on the latter giving those doubly tangent to $c^{2}$. If $c^{2}$ happens to be on a quadric surface $Q$ of the web, $F^{6}$ is a tangent cone of $F^{3}$ having four nodal and six cuspidal elements and its vertex corresponds to $Q$. In either case, $F^{6}$ is tangent to $\Delta_{2}{ }^{4}$ at 8 points at which $\gamma^{6}$ is tangent to $\Theta_{1}{ }^{6}$, the curve of contact between $F^{3}$ and $\Delta_{2}{ }^{4}$. These 8 points correspond to the 8 points in which $c^{2}$ meets $J_{2}^{4}$. Now the two surfaces, $F^{6}$ and $F^{3}$, intersect in another sextic curve and the points of this curve yield the quadric surfaces of the web which are tangent both to $f$ and to $c^{2}$.

Finally, let a point $P$ be given in $f$. The quadric surfaces of the web passing through $P$ form a net and they are given by the points of a plane $\pi$ in $\Sigma_{3}$. If $P$ is on a fixed line $l$ of $f, \pi$ is tangent to $F^{2}$ along a line which is tangent to $\Theta_{1}{ }^{3}$ at one point. $\pi$ is tangent to $F^{3}$ at the same point. There are $\infty^{1}$ cones of the web passing through $P$ and to these cones correspond the points of the quartic curve in which $\pi$ meets $\Delta_{2}{ }^{4}$. The vertices of these cones lie on a sextic curve of $J_{2}{ }^{4}$.

University of California, Berkeley, Calif. 